DOI: $10.21105 /$ joss. 00058

\section{Software}

- Review ¿

- Repository ca

- Archive ${ }^{\top}$

\section{Licence}

Authors of JOSS papers retain copyright and release the work under a Creative Commons Attribution 4.0 International License (CC-BY).

\title{
SEP: Source Extractor as a library
}

\section{Kyle Barbary ${ }^{1}$}

1 University of California, Berkeley

\section{Summary}

Source Extractor (Bertin and Arnouts 1996; Bertin 2016) is a widely used command-line program for segmentation and analysis of astronomical images. It reads in FITS format files, performs a configurable series of tasks, including background estimation, source detection, deblending and a wide array of source measurements, and finally outputs a FITS format catalog file.

While Source Extractor is highly useful, the fact that it can only be used as an executable - reading input files, producing output files and controlled by a limited set of configuration options specified in another file - can limit its applicability or lead to awkward workflows. There is often a desire to have programmatic access to perform one or more of the above tasks on in-memory images as part of a larger custom analysis.

SEP makes available the core algorithms of Source Extractor in a library of stand-alone functions and classes. These operate directly on in-memory arrays (no FITS files or configuration files). The code is derived from the Source Extractor code base (written in C) and aims to produce results compatible with Source Extractor whenever possible. SEP consists of a $\mathrm{C}$ library with no dependencies outside the standard library, and a Python module that wraps the $\mathrm{C}$ library in a Pythonic API. The Python wrapper operates on NumPy arrays with NumPy as its only dependency. It is generated using Cython.

From Source Extractor, SEP includes background estimation, image segmentation (including on-the-fly filtering and source deblending), aperture photometry in circular and elliptical apertures, and source measurements such as Kron radius, "windowed" position fitting, and half-light radius. Additionally, several features not in Source Extractor have been added:

- Optimized matched filter for variable noise in source extraction.

- Circular annulus and elliptical annulus aperture photometry functions.

- Local background subtraction in shape consistent with aperture in aperture photometry functions.

- Exact pixel overlap mode in all aperture photometry functions.

- Masking of elliptical regions on images.

Finally, note that SEP is essentially a fork of Source Extractor that has already diverged significantly from the original code base. One might ask why SEP is not part of Source Extractor itself: the command-line interface in Source Extractor could be built on top of such a library. The answer is that a vast array of changes were necessary in order to expose the functionality as stand-alone $\mathrm{C}$ functions. It would be a lot of work to rewrite Source Extractor itself in this way, with little gain for the executable itself. 


\section{References}

Bertin, E., and S. Arnouts. 1996. "SExtractor: Software for source extraction." 117 (June): 393-404. doi:10.1051/aas:1996164.

Bertin, Emanuel. 2016. "SExtractor." http://www.astromatic.net/software/sextractor. 